\title{
A RECENT MACHINE LEARNING TECHNIQUES FOR FAILURE DIAGNOSIS OF ROLLING ELEMENT BEARING
}

Author(s):

M. H. Albdery ${ }^{1,2}$, I. Szabó ${ }^{1}$

Affiliation:

${ }^{1}$ Institute of Technology, Hungarian University of Agriculture and Life Science (MATE)

2 Doctoral School of Mechanical Engineering, Institute of Technology, Hungarian University of Agriculture and Life Science (MATE), 2100 Gödöllö, Páter Károly u. 1., Hungary

\section{Email address:}

mohsinhassaniq@gmail.com; Szabo.Istvan.prof@uni-mate.hu

\begin{abstract}
Rolling element bearings are critical components of rotating machines, and fault in the bearing can cause the machine to fail. Bearing failure is one of the leading causes of failure in various rotating machines used in industry at high and low speeds. Fault diagnosis of various rotating equipment plays a significant role in industries as it guarantees safety, reliability and prevents breakdown and loss of any source of energy. Early identification is an essential element in the diagnosis of defects that saves time and expenses and avoids dangerous conditions. Investigations are being carried out for intelligent fault diagnosis using machine learning approaches. This article gives a short overview of recent trends in the use of machine learning for fault detection. Finally, Deep Learning techniques were recently developed to monitor the health of the intelligent machine are discussed.
\end{abstract}

Keywords: machine learning, rolling element bearing, failure detection, artificial intelligent method, deep learning

\section{Introduction}

Rotating machines are widely used in industrial environments because of their cost-effectiveness, performance, and durability. They are frequently exposed to harsh working environments such as higher load, higher speed, and restricted lubrication. Rolling element bearings are the most vulnerable component of a machine. However, they are frequently operated in harsh and hazardous conditions, resulting in component failure during operation, jeopardizing worker safety and resulting in economic loss. Over $42 \%$ of mechanical failures are due to bearing failure (Singh et al., 2019).

According to Heng (2009) the primary cause of mechanical failure was bearing failure, which resulted in an increase in warranty and maintenance costs. In some instances, bearing failures can result in the total failure of the machinery. This has risen to prominence as a critical subject area as a result of the ease with which the health of rolling element bearings can be determined using specific techniques such as machine learning. The vibration signature reveals, there is a symptom indicating early that information, it serves as a crucial indication of a problem within them. The main failure reasons of rolling element bearing are imbalance shaft faults, ball bearing defects, inner race faults, outer race faults, and cage faults.

A bearing consists of an inner, outer race and rollers, but in many cases is made up of more than one type of each of these. The geometry of the bearing can produce a unique frequency for each bearing. The outer race of rolling element bearing is typically stationary, with most of its faults occurring in the load zone. As a result, the defect impulses will not be modulated in the manner depicted in Figure 1. In comparison, the defect impulses for inner race faults and rolling elements are modulated at shaft frequency and FTF as they pass through the load zone (Robert B Randall, 2004). Additionally, even harmonics of the BSF are frequently dominant, as the rolling element's fault engages the inner and outer races once per revolution.

The traditional methods of detecting the presence of a bearing fault solely rely on the frequency characteristics. When we examine the data signals closely, we notice that they contain a hidden pattern that is difficult for humans to identify. As a result, researchers began developing machine learning algorithms 


\section{BEARING}

such as ANN, KNN, and SVM. These algorithms analyse the data pattern and make an intelligent determination regarding the presence of a bearing fault. The majority of these algorithms produce satisfactory results in classifying bearing faults with greater than $90 \%$ accuracy. Over the last five years, deep learning techniques have been used to improve and increase the accuracy of fault classification.

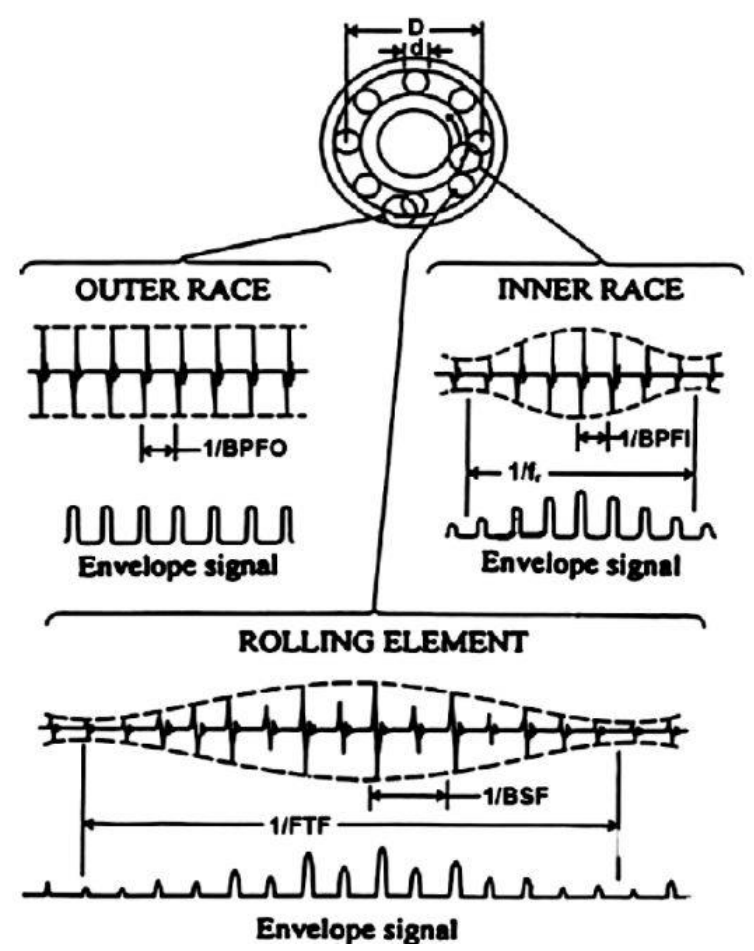

Figure 1. Rolling element bearing vibration signal characteristics due to local faults (Robert B Randall, 2004)

The purpose of this article is to provide an overview of recent trends in research on rolling element bearing failure diagnosis by using machine learning techniques and also Deep Learning techniques and their benefits.

\section{Modes of Bearing Failure}

Different factors such as cracks, mechanical damage, wear and tear, corrosion and insufficient lubrication can cause faulty bearings. The components of the coating are progressively wear deteriorated. As a result of poor lubrication, friction between the contact surfaces increases, leading to increased bearing element temperature (Heng et al., 2009). In the following, most of the failure modes occur in roller bearing as demonstrated Figure2:

1. Fatigue:

It starts with a small crack on the bearing surface (rollers or races) due to a change in the material structure caused by repeated stress in the contact areas.

2. Wear:

Wear is produced by dirt or foreign particles inside the rolling in or against the seal rollers because of a deficiency of lubrication or compression.

3. Electric erosion:

It is damage (in the form of craters) to one of the bearing components (rollers or races) caused by an electric current passing through the bearing.

4. Corrosion:

Corrosion occurs when water or other corrosive agents enter the bearing through damaged seals, acidic lubricants, or a rapid change in operating temperature.

5. Plastic deformation:

Plastic deformation occurs primarily when the bearing is subjected to an excessive load, causing the raceways to indent. 


\section{Fracture and cracking:}

Fracture and cracking are the results of stress caused by abrasive treatment (impacts) or cyclic stress. Additionally, high heating can result in fracture and cracking.

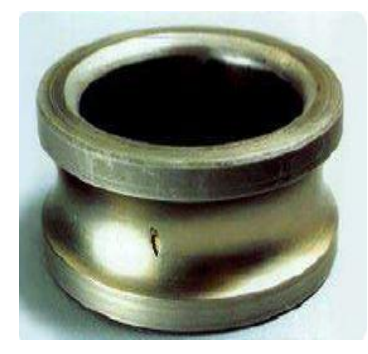

a. Fatigue

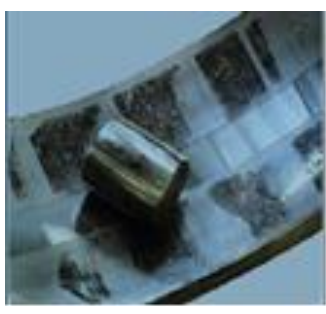

d. Corrosion

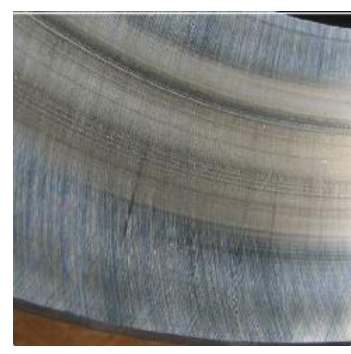

b. Wear

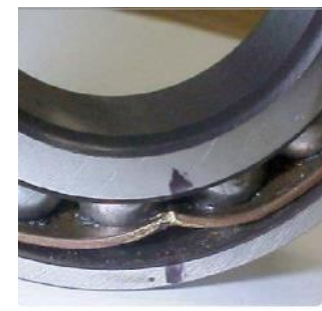

e. Plastic deformation

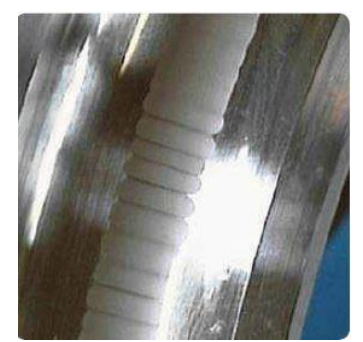

c. Electrical erosion

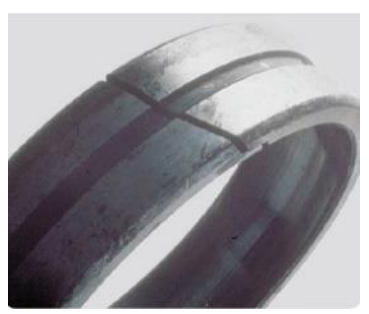

f. Fracture

Figure 2. Modes of failure of rolling element bearing (Chen and Li, 2017).

\section{Machine Learning (ML) Techniques for Failure Diagnosis:}

Bearing fault diagnosis uses machine learning and artificial intelligence (AI) to improve the CM system and, as a result, the rotating machine's reliability. A wide range of algorithms are available in machine learning, and the algorithms are chosen based on the application (Raúl et al., 2019). There are three distinct types of machine learning algorithms: supervised, unsupervised, and semi-supervised. Supervised learning entails inputting known data and evaluating it using probability (Jiaying et al., 2019). In contrast, the input data are not known in unsupervised learning, and the algorithm is intended to detect data structures. For semisupervised learning, input data are an input function of the combination of labelled and unlabelled values, with evaluation being conducted. The application of ML for the purpose of diagnosis and prediction in other fields are studied (Lijun et al., 2018).

Many different types of traditional ML methods are available, including ANN, SVM, Decision Tree, KMeans, KNN and Random Fault Diagnostic Forest Algorithm. The data must be processed with the Feature Engineering and Feature Extraction when the dimension of the data needs to be reduced and the main component analysis is used before transmitting the data to the classification algorithm (Awadallah et al., 2003).

Early detection of incipient defects has shown some research that AIs such as ANN, fuzzy and adaptive fuzzy can be detected (Filippetti et al., 2000) for electrical engines, with characteristics such as frequency spectrums investigated.

\subsection{Artificial Neural Network (ANN)}

Artificial neural networks (ANN) have recently gotten a lot of attention in the industrial world. Artificial neural networks (ANNs) are supervised ML algorithms competent enough to solve problems such as pattern detection, clustering, classification, regression and nonlinear functional estimation (Jammu et al., 2011). Additionally, ANN is used to process and classify data. Similarly, Jia et al., (2016) presents an AI selfadaptive FDD system inspired by genetic algorithms (GA) and nearest neighbour (NN). To find 
approximation coefficients during the feature extraction stage, a two-dimensional discrete wavelet transform (2D-DWT) is used in conjunction with Shannon entropy. Additionally, GA and nearest neighbour techniques are used to determine the histograms of selected coefficients for use as inputs to the feature space selection method. The cost-effectiveness, non-contact, and non-intrusive nature of this method is the primary advantages. The multilayer perceptron (MLP) is a supervised learning neural network with multiple layers (Lei et al., 2011). Wenyi et al., (2011) is describe an approach for identifying FDD bearing faults using ANN for IM. Additionally, the proposed pattern identification approach makes use of two current sensors. Thus, a multilayer perceptron (MLP) is used, which has one and two hidden layers (Li et al., 2019).

Nerella et al., (2018) developed an ANN model to predict the size of defects in cylindrical roller bearings (N312). It was discovered that the experimental data and predicted values for the AE level are highly correlated (6.90 percent of error). Gunerkar et al., (2019) investigated ball bearing ANN and KNN classification of faults using successfully trained and tested wavelet transform data. Five significant features were fed into the ANN and KNN models as inputs. The proposed ANN model demonstrated exceptional efficacy in classifying the multiple faults described in the second fault class. As a result, such an ANN model can be used to diagnose multiple faults and as a novel approach to a particular diagnostic problem.

\subsection{Support Vector Machine (SVM)}

It frequently combines the wavelet method, empirical mode decomposition (Jiaying et al., 2019; Hinton et al., 2006), and spectral analysis (Lijun et al., 2018) to determine the time domain and frequency domain. The statistical properties of vibration signals, such as root mean square value, kurtosis, power spectral density, information entropy and sideband index, as the main identification targets. Each Statistical characteristics of vibration signals can be used to infer their overall characteristics ( $\mathrm{Li}$ et al., 2019). However, the local information contained in the signal data will be masked by the statistical features, which cannot accurately reflect the signal's local details. The misclassification ratio is higher for problems involving multiple classifications and insensitive fault features. When dealing with large amounts of data, it's all too easy to fall victim to the disaster of dimensionality. Chen et al. (2021) propose a method for diagnosing rolling bearing faults based on refined composite multiscale fuzzy entropy (RCMFE), topology learning and out-of-sample embedding (TLOE), and a marine predators algorithm based on support vector machines (MPA-SVM). The proposed method was validated using data from Case Western Reserve University and fault diagnosis experiments performed on 1210 self-aligning ball bearings. The results demonstrate the efficacy of the fault diagnosis method, which is capable of diagnosing bearing faults with an accuracy of up to 100 percent.

\subsection{Combined ANN and SVM}

To achieve high diagnostic performance, it has been proposed to combine ANN and SVM techniques. More precisely, Yu et al., (2019) proposes an fault detection approach for rolling element bearings that is based on the extraction of statistical features from vibration signals. For this purpose, the statistical characteristics are derived using advanced signal processing techniques and central limit theory.

Notably, the output feature vector (statistical feature vector) is used as an input vector for a classifier that uses ANN and SVM to categorize various types of faults. As a result, the authors argued in this study that ANN and SVM could not provide an analytical guarantee for the FDD classifier's accuracy. Additionally, Bertolini et al., (2021) introduces an fault detection method for ball bearings that incorporates both ANN and SVM. Additionally, statistical techniques are used to reconstruct the time domain characteristics of vibration signals. Following this, the classification stage employs ANN and SVM (Li et al., 2020). Kankar et al. (2011) investigated ball bearing fault diagnosis using machine learning methods, the purpose of this research is to determine the cause of ball bearing failures using an artificial neural network (ANN) and a support vector machine (SVM). The results indicate that machine learning algorithms can be used to perform automated bearing fault diagnosis. It was concluded that SVM is more accurate than ANN.

\subsection{KNN Based algorithm (kNN)}

K-Nearest neighbour ( $\mathrm{kNN}$ ) algorithm is a classification algorithm in which the data are divided into various categories to predict the classification. David He et al., (2011) carried out a full detection of ceramic bearings using acoustic emission-based condition indicators (CI). Classification accuracy was improved by merging 
all individual CI's, with this method more than 92\% accuracy was accomplished. M. M. Ettefagh et al., (2020) a hybrid method was applied in association with "Genetic Algorithm" (GA) and K-Means clustering was used to find the fault in bearing, it was found that GA-K means algorithm gives better performance than traditional K-means. Jing Tian et al. et al., (2016) fault diagnosis of REB of electric motor was carried out. The extracted vibration signals are developed with the help of spectral kurtosis and cross- correlation, firstly decomposing the frequency domain to sub-signal, if the signals are faulty then envelope analysis is performed to detect four faulty signatures associated with IR, OR, rolling element and cage faulty that would be cited as a base reference signals, if the bearing comes under in any of these reference signals it would identify the signals as faulty and also hidden faults were able to be identified with this method. Thomas W. Rauber et al. (2015) have stated that KNN Based algorithm bearing fault diagnosis technique restricts the process summary to single feature model i.e. not more than one contemporaneous feature model is considered. Hence in this paper, sequence of several feature models with feature selection method is applied that will enhance the classification performance. A process description is carried out extensively and a simple k-NN classifier was able to produce the results. Wang et al., (2020) propose a KNN-based method for real-time online fault diagnosis of rolling bearings. The method is divided into two stages: model training for fault diagnosis and real-time online fault diagnosis. To begin, the vibration signal is pre-processed: classification, cleaning, segmentation, and feature parameter extraction are performed, followed by training and optimization of the fault diagnosis model. The results indicate that the fault diagnosis model based on KNN algorithm outperforms the fault diagnosis models based on C4.5 and CART algorithms, indicating that the fault diagnosis model based on KNN algorithm is more suitable for rolling bearing fault diagnosis. Utilizing this method to diagnose rolling bearings enables predictive maintenance prior to bearing failure and minimizes economic losses associated with unplanned downtime of critical equipment.

\subsection{Deep learning diagnosis Techniques}

Deep learning (Hinton al., 2006) basically refers to a class of ML techniques, where many layers of information processing stages in deep architectures are exploited for pattern classification and other tasks (Jia F et al., 2016) Deep learning has the potential to overcome the inherent deficiencies of traditional intelligent methods. It has the ability to adaptively capture sensitive fault information and automatically learn valuable fault features from raw data through multiple nonlinear transformations and approximate complex nonlinear functions with a small error. Thereby, they do not only get rid of manual feature extraction but also learn complex nonlinear relationships with ease. A high-level illustration of the basic differences between the conventional ANN and deep learning approach is depicted in figure 16. Deep learning models have several variants Schmidhuber (2015) such as deep auto-encoders (Rauber et al., 2015), deep belief networks (DBMs) (Hinton et al., 2006), convolutional neural networks (CNN) (LeCun et al., 1998) and recurrent neural networks (RNNs) (Funahashi et al., 1993). Zhao et al., (2021) proposed a deep adversarial network with joint distribution adaptation for diagnosing rolling bearing transfer faults. To effectively address the aforementioned fault diagnosis issues, a joint distribution adaptation network with adversarial learning is developed. To begin, they construct a deep convolutional neural network (CNN) to extract features from training and test samples. Second, because the joint maximum mean discrepancy (JMMD) cannot accurately quantify the joint distribution discrepancy between different domains, an improved joint maximum mean discrepancy (IJMMD) is proposed to match the feature distributions the proposed method is capable of accurately matching distributions and extracting category discriminative and domain-invariant features shared by the source and target domains.

Shenfiel et al., (2020) develop an intelligent fault diagnosis method capable of operating on these real-time data streams to provide early detection of developing problems under variable operating conditions. They propose a novel dual-path recurrent neural network with a wide first kernel and deep convolutional neural network pathway (RNN-WDCNN) capable of operating on raw temporal signals such as vibration data to diagnose rolling element bearing faults in data acquired from electromechanical drive systems.

\subsubsection{Convolutional neural network (CNN)}

One deep learning technique is the convolutional neural network (CNN) approach. $\mathrm{CNN}$ is a feed forward neural network of multiple layers, which assumes inputs as images (S. Min et al., 2017). It was inspired by neurons of the human visual cortex that have two features (Y. LeCun et al., 2015). One is local connections, 
which means that since images have high correlation within sub-regions, the correlation information is critical in recognizing those images, where the subregions in the previous layer are connected to local patches in the feature maps by filters. The other feature is shared weights, where a pattern can appear in various locations in the images, and by convoluting filters across an image, the pattern can be extracted independent of location. In addition, using the same filter across an image, the number of parameters is reduced significantly. Nowadays, many open-sourced CNN models are available (e.g., GoogLeNet, AlexNet) which make them attractive to researchers. $\mathrm{CNN}$ is structured by a series of layers, in which the convolutional and pooling layers come first, and the fully connected layers come last. A descriptive example of the CNN architecture is shown in Figure 3. The convolutional layer is used to detect local correlation from the previous layer (the raw input). It has a number of hyper-parameters, such as the number of filters, the filter size and the stride. Wang et al. (2020) propose a method for implementing CNN in fault classification, this method compresses the time-domain vibration signals of multiple sensors located in different locations into a rectangular two-dimensional matrix and then classifies the signals using an improved two-dimensional CNN. The method was validated using open datasets from Case Western Reserve University, the University of Cincinnati's IMS bearing database, and a dataset from a custom-built bearing fault test rig. It achieved 99.92 percent, 99.68 percent, and 99.25 percent prediction accuracy, respectively.

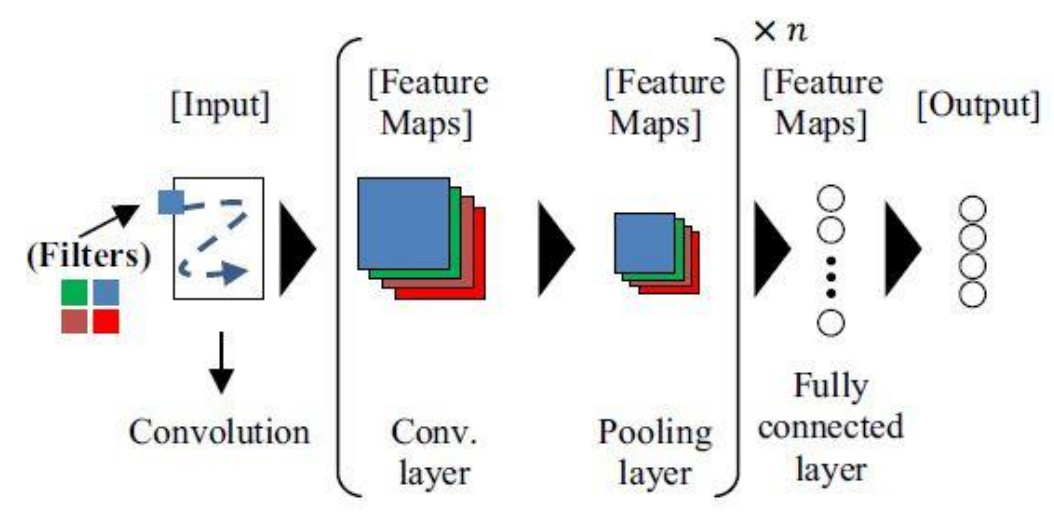

Figure 3. Descriptive example of convolutional neural network (CNN)

\subsubsection{Deep belief network}

The structure of deep belief networks (DBNs) is composed of a stacked network of restricted Boltzmann machines (RBMs). Each RBM consists of a visible layer (input), a hidden layer and an input layer. The schematic architecture of an RBM consist from $v$ denotes the input layer and $h 1, h 2$ and $h 3$ are the hidden layers. $v$ and $h 1$ constitute the first RBM (RBM 1), h1 and h2 constitute the second RBM (RBM 2) and h2 and $\mathrm{h} 3$ constitute the third RBM (RBM 3). DBNs are trained in two stages: individual training of the RBM layers step by step in a greedy way, and then, fine-tuning the whole network (parameter adjustment) to achieve an ideal performance (Zhang $M$ et al., 2011). The greedy layer-wise training is a pre-training algorithm that aims to train each layer of a DBN in a sequential way, feeding lower layers' results to the upper layers. Chen et al (2017) proposed a multi-sensor feature fusion technique where two-layer SAEs were used for feature fusion and a three-layer RBM-based DBN was used for classification. Since then, there have been many attractive implementations and uses of DBN in the domain of PHM of REBs. DBNs (typically stacked RBMs) can be used both for classification and as well regression (Xia M et al., 2017), which is an extended form of multi-layer RBM with structures of an FFNN. Shao et al (2015) developed a DBN-based REB fault diagnosis system incorporated with particle swarm optimization. In another work Shao et al (2017), they proposed a dual-tree complex wavelet package to extract fault features and DBN was used to classify multiple types of bearing faults. Gan et al (2016) developed a hieratical structure of bearing diagnosis using ensemble DBN. In the first level, four types of fault- s/health were classified on two layers of DBN, and another two layers of DBNs were designed for fault severity identification. With regard to prognostic problems, Deutsch et al (2017), predicted the remaining life of bearings using DBN with a particle filter. Zhang et al (2017) proposed a multi- objective evolutionary algorithm integrated with DBNs for bearing remaining life prediction, aiming to optimize the accuracy and generalization performance of the DBN 
simultaneously. Ma et al (2017) used ant colony optimization to determine the structure of the DBN and then the DBN was used to predict the health status of a machine, specifically on bearing components. Some works used DBN as a feature learning tool embedded into a fault diagnosis structure. Xu et al (2019) applied three layers of RBMs to automatically extract features from raw signals and then used PCA to shrink features and implemented independent classification models for fault diagnosis. Zhang et al. (2020) presented an enhanced CNN model for bearing fault diagnosis that utilized time-frequency images as inputs. Seven datasets provided by CWRU and YSU were used to validate the proposed method's efficacy. As demonstrated by the diagnosis results, the proposed method extracts sensitive features much more rapidly and accurately than existing methods on small data sets with sample sizes of 200 and sample lengths of 512. Additionally, when the workload, such as dataset F, changes, the performance of time domain and frequency domain theory rapidly degrades. However, the proposed method is highly adaptable to changes in workload.

\subsubsection{Auto-encoders}

An auto-encoder is a three-layer neural network that is trained to minimize its input to its output (minimize reconstruction error between input and out- put). It consists of two parts: an encoder and a decoder. The encoding operation maps input to a hidden layer of neurons and the decoding operation reconstructs the input from the hidden layer. Auto-encoders are purely used for unsupervised feature extraction as they can be trained more easily and effectively. The structure of a standard auto-encoder includes an input layer, a hidden layer and an output layer. Several auto-encoders when stacked together to form a deep structure to learn representations by taking the output of each hidden layer (ith) as an input to train the next $(i+1)$ layer; this architecture is known as a deep auto-encoder (DAE). One of the earliest implementations of auto-encoders in the PHM of REBs can be found in it, where a five- layer auto-encoder-based deep neural network (DNN) was utilized to classify the health conditions of the machinery. It was observed that the classification performance of DNNs (99.6\%) significantly outperformed that of backpropagation- based neural networks (70\%). Thereafter, typical DAE-based approaches have generally focused on auto-feature representation (mapping to a higher dimension) and sensor fusion (mapping to a lower dimension). Adding a fully connected layer and soft max layer, it can both used for classification and regression. Jia et al (2016) validated the feasibility of implementation of DAE in REB fault diagnosis. Lu et al (2015) developed a health state identification model using a stacked denoising auto-encoder. Meng et al (2018) applied a denoising autoencoder on raw signals and proposed an active regularization parameter tuning strategy. Regularization hyperparameters can be automatically tuned by increasing the number of layers. Jia et al (2018) designed a local connection network constructed by a normalized sparse auto-encoder to deal with fault signal shifting among different working conditions. Some other works which employ auto-encoder in bearing fault diagnosis using the CWRU dataset. Chen et al., (2020) examined three deep neural network models (Deep Boltzmann Machines, Deep Belief Networks, and Stacked Auto-Encoders) for identifying rolling bearing fault conditions. Four pre-processing schemes are discussed, including those in the time domain, frequency domain, and time-frequency domain. A single data set containing seven fault patterns is used to assess the performance of deep learning models for diagnosing rolling bearing faults based on the health state of a rotating mechanical system. The results demonstrate that the accuracy achieved by Deep Boltzmann Machines, Deep BeliefNetworks, and Stacked Auto-Encoders is extremely reliable and applicable to rolling bearing fault diagnosis.

\subsubsection{Recurrent Neural Network (RNN)}

RNN is a type of neural network that contains loops, enabling information to be stored within the system. Feature extraction step was carried out with the help of a discrete wavelet transform and for best classification accuracy orthogonal fuzzy neighbourhood discriminant analysis (OFNDA) was used by Wathiq et al (2015) as shown in Figure 4. After this step the features are passed onto RNN method for fault classification which would detect and classify faults under dynamic operating condition. Overall classification accuracy for the proposed method was found to be $97 \%$. Honghu Pan et al. (2018) have proposed a method combining one dimensional convolutional and LSTM that are constructed into one framework.

The data were fed to CNN model which reduces the frequency variance and the result of the CNN were supplied to the input of the LSTM which has the advantage of the temporal model. Comparing with the traditional methods, proposed methods have the highest average accuracy rate prediction of $99 \%$. Liang Guo 
et al. (2017), have intended to study the RUL of a bearing using RNN- Health Indicators (RNN-HI) to increase the RUL Prognostic accuracy. The mapping of the time and frequency domain features for the extraction process were performed, obtained characteristics were chosen based on specific criteria metric. These characteristics were fed to the RNN and subsequently to RNN-HI so to build the proposed model. The proposed model was compared with self-organizing map (SOM) and this method performs better than HISOM. Hongkai Jiang et al. (2018), a "deep recurrent neural network" (DRNN) was obtained by stacking a number of recurrent neural network which captures the features automatically from the input data. In the next step adaptive learning method was implemented for training samples with the constructed DRNN and then finally the testing samples were checked for the proposed method. The average training and testing accuracy of $98.67 \%$ and $96.53 \%$ respectively. An et al., (2020) proposed a three-part model that is capable of ignoring the effect of varying rotational speeds. To begin, the sample is segmented, and each segment dimension is extended via an input network to ensure sufficient memory space for the information. Second, classification information is stored and transferred in a long short-term memory (LSTM) network before being output to the third section. Due to the gate units' function, the working condition information is ignored during this process. The proposed method is validated using bearing datasets with time-varying speeds and loads. The result indicates that our method is more accurate with a simpler structure and outperforms the traditional method in diagnosing bearing faults.

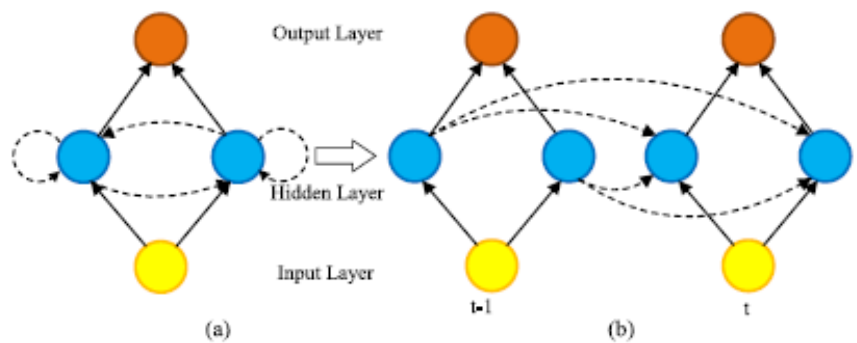

Figure 4. (a) Construction of RNN and (b) RNN over a time period (Wathiq et al 2015)

\subsubsection{Generative Adversarial Network (GAN)}

Generative adversarial networks are mathematical design that uses couple of neural networks, indenting one against the other (therefore the "adversarial" name) in order to generate new real data. Han Liu et al (2018) have studied a unique deep neural network called Categorical adversarial auto encoder (CatAAE) which enforces a previous allocation to latent coding space for unsupervised learning. The encoder compensated for producing examples to fool the classifier and later a classifier was trained to distinguish earlier division from the counterfeit division. In the course of adversarial training process, the identical faults have been allocated to the similar groups of classifiers. This proposed method was compared with conventional KMeans technique and the result showed better accuracy with the proposed method for Y. O. Lee et al. (2017) The discriminator classifies and differentiates the real and counterfeit data and later generator oversample the data generator which would be difficult to be classified by the discriminator. Authors have shown achievability of resolving the challenges associated with data imbalance using the proposed method. Yuan Xie et al. (2018) used a new technique to overcome the imbalanced dataset using deep convolutional generative adversarial network model for detection of faults in bearings. The original training data are fed to DCGAN, where it deals with the minority classes. Also "synthetic minority oversampling technique" (SMOTE) was used where it generates synthetic minority samples to balance the dataset. For classification of the fault, a SVM technique is used to verify better efficiency of the generative paradigm. The proposed paradigm showed in Figure 5 an accuracy for training and testing sample to be $95.64 \%$ and $86.33 \%$ respectively.

Bo Zhang et al. (2018) have generated a novel on adversarial adaptive 1-D CNN (A2CNN) based on DNN for the fault diagnosis of REB. Firstly, label classification error is minimized by labelling the source samples for source feature extractor. And then maximization of domain classification loss was attained by using target feature extractor to make sure that the source and target features have identical distribution following 
mapping. Efficiency of the model was measured by the precision and recall parameters. S. Suh et al. (2019), have proposed a unique over sampling method to resolve the difficulties of data imbalance for the diagnosis of a bearing. This method was introduced because usually faulty data is lower compared to normal data. Firstly time-series data was converted into image domain using nested scalar plot (NSP) and then oversampling method Wasserstein GAN with gradient penalty on Deep convolution generative adversarial networks (DCWGAN-GP) used to overcome the data imbalance and then the CNN method was used classify the bearing faults. The proposed method showed better accuracy after implantation of the proposed model. The proposed classifier accuracy got improvised from $88 \%$ to $99 \%$.

Viola et al., (2021) propose the FaultFace methodology for detecting failures in Ball-Bearing joints for rotational shafts by utilizing deep learning techniques to generate balanced datasets. The Fault Face methodology makes use of two-dimensional representations of vibration signals dubbed face portraits that are created using time-frequency transformation techniques. To achieve a balanced dataset, a Deep Convolutional Generative Adversarial Network is used to generate new face-portraits of the nominal and failure behaviours from the obtained face-portraits. A balanced dataset is used to train a Convolutional Neural Network for fault detection. The Fault Face methodology is compared to other deep learning techniques to determine its performance when used with unbalanced datasets for fault detection. The obtained results demonstrate that the Fault Face methodology is effective at detecting failures in unbalanced datasets.

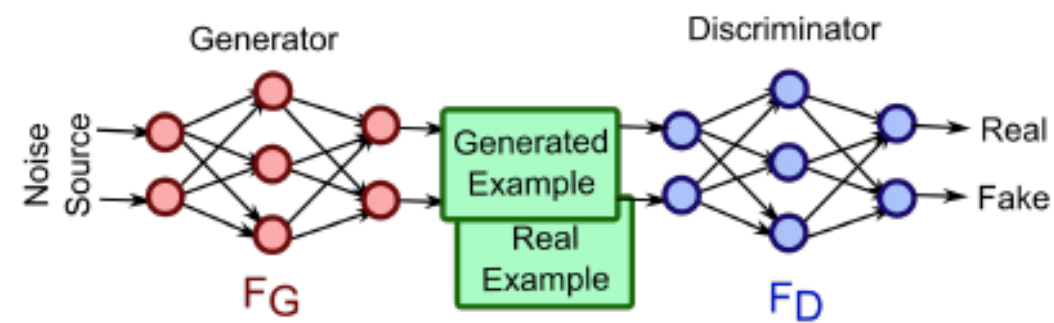

Figure 5. Construction of Generative Adversarial Network (GAN) (Xie et al., 2018)

\section{Conclusion}

The purpose of this article is to provide a general descriptive overview of the machine learning techniques for failure detection currently used in rolling element bearing diagnostics for rotating machinery. Machine learning techniques are discussed in along with its advantages and disadvantages. Numerous researchers have also demonstrated that using ANN Deep learning diagnosis Techniques can detect bearing faults at a high level. Although traditional techniques produce acceptable results, they require feature engineering and feature extraction prior to classification, and subject expertise is required to perform feature engineering and feature extraction for traditional techniques. Whereas deep learning requires a larger dataset to train the sample data and has numerous advantages since it automatically performs feature engineering and feature extraction without the need for subject field expertise. These techniques can detect bearing faults more quickly and with better accuracy.

\section{Acknowledgement}

Special thanks to the the Stipendium Hungaricum Scholarship Programme, Institute of Technology and the Mechanical Engineering Doctoral School, Hungarian University of Agriculture and Life Sciences, Gödöllö, Hungary.

\section{References}

[1] An, Z., Li, S., Wang, J., \& Jiang, X. (2020). A novel bearing intelligent fault diagnosis framework under time-varying working conditions using recurrent neural network. ISA transactions, 100, 155170.

[2] Bengio, Y., Courville, A., \& Vincent, P. (2013). Representation learning: A review and new perspectives. IEEE transactions on pattern analysis and machine intelligence, 35(8), 1798-1828. 
[3] Bertolini, M., Mezzogori, D., Neroni, M., \& Zammori, F. (2021). Machine Learning for industrial applications: a comprehensive literature review. Expert Systems with Applications, 114820.

[4] Chen Z. and Li W. (2017). Multisensor feature fusion for bearing fault diagnosis using sparse autoencoder and deep belief network IEEE Trans. Instrum. Meas. 66 1693-702

[5] Chen, X., Qi, X., Wang, Z., Cui, C., Wu, B., \& Yang, Y. (2021). Fault diagnosis of rolling bearing using marine predators algorithm-based support vector machine and topology learning and out-ofsample embedding. Measurement, 176, 109116.

[6] Chen, Z., Deng, S., Chen, X., Li, C., Sanchez, R. V., \& Qin, H. (2017). Deep neural networks-based rolling bearing fault diagnosis. Microelectronics Reliability, 75, 327-333.

[7] D. He, R. Li, J. Zhu, and M. Zade "Data mining based full ceramic bearing fault diagnostic system using AE sensors," IEEE Trans. Neural Networks, vol. 22, No. 12 PART 1, pp. 2022-2031, 2011, doi: 10.1109/TNN.2011.2169087.

[8] Deutsch J. and He D. (2018). Using deep learning-based approach to predict remaining useful life of rotating components IEEE Trans. Syst. Man Cybern. Syst. 48 11-20

[9] Deutsch J., He M. and He D. (2017). Remaining useful life prediction of hybrid ceramic bearings using an integrated deep learning and particle filter approach Appl. Sci. 7649

[10] F. A. Chaves and D. Jiménez, (2018). Intelligent fault diagnosis of rolling bearing using improved deep recurrent neural network," Nanotechnology, vol. 29, No. 27,

[11] F. Filippetti, G. Franceschini, C. Tassoni, S. Member, and P. Vas, (2000). Recent Developments of Induction Motor Drives Fault Diagnosis Using AI Techniques. IEEE Trans. Ind. Electron., vol. 47, no. 5, pp. 994-1004.

[12] Funahashi K and Nakamura Y. (1993). Approximation of dynamical systems by continuous time recurrent neural networks Neural Netw. 6 801-6

[13] Gan M. and Wang C. (2016). Construction of hierarchical diagnosis network based on deep learning and its application in the fault pattern recognition of rolling element bearings Mech. Syst. Signal Process. 72 92-104

[14] Gunerkar, R. S., Jalan, A. K., \& Belgamwar, S. U. (2019). Fault diagnosis of rolling element bearing based on artificial neural network. Journal of Mechanical Science and Technology, 33(2), 505-511.

[15] H. Liu, J. Zhou, Y. Xu, Y. Zheng, X. Peng, and W. Jiang, (2018). Unsupervised fault diagnosis of rolling bearings using a deep neural network based on generative adversarial networks," Neurocomputing, vol. 315, pp. 412-424, doi: 10.1016/j.neucom.2018.07.034.

[16] H. Pan, X. He, S. Tang, and F. Meng, (2018). An improved bearing fault diagnosis method using one-dimensional CNN and LSTM. J. Mech. Eng., vol. 64, no. 7-8, pp. 443-452, doi: 10.5545/svime.2018.5249.

[17] Heng A, Zhang S, Tan A C C and Mathew J (2009). Rotating machinery prognostics: state of the art, challenges and opportunities Mech. Syst. Signal Process. 23 724-39

[18] Hinton G E and Salakhutdinov R. R. (2006). Reducing the dimensionality of data with neural networks Science (80-.) 313 504-7

[19] Hinton G E, Osindero S and Teh Y-W (2006). A fast learning algorithm for deep belief nets Neural Comput. 18 1527-54

[20] Hinton G. E. and Zemel R. S. (1994). Autoencoders, minimum description length and Helmholtz free energy Adv. Neural Inf. Process. Syst. (NIPS 1994) vol 7 (Cambridge, MA: MIT Press) 3-10

[21] J. Tian, C. Morillo, M. H. Azarian, and M. Pecht (2016). Motor Bearing Fault Detection Using SpectralKurtosis-Based Feature Extraction Coupled With K -Nearest Neighbor Distance Analysis. IEEE Trans. Ind. Electron., vol. 63, no. 3, pp. 1793-1803,.

[22] Jammu, N. S., \& Kankar, P. K. (2011). A review on prognosis of rolling element bearings. International Journal of Engineering Science and Technology, 3(10), 7497-7503.

[23] Jia F., Lei Y., Guo L, Lin J. and Xing S. (2018). A neural network constructed by deep learning technique and its application.

[24] Jia, F., Lei, Y., Lin, J., Zhou, X., \& Lu, N. (2016). Deep neural networks: A promising tool for fault characteristic mining and intelligent diagnosis of rotating machinery with massive data. Mechanical Systems and Signal Processing, 72, 303-315.

[25] Jiaying, D.; Wenhai, Z.; Xiaomei, Y. (2019). Recognition and Classification of Incipient Cable Failures Based onVariational Mode Decomposition and a Convolutional Neural Network. Energies, $12,2005$. 
[26] Kankar, P. K., Sharma, S. C., \& Harsha, S. P. (2011). Fault diagnosis of ball bearings using machine learning methods. Expert Systems with applications, 38(3), 1876-1886.

[27] L. Guo, N. Li, F. Jia, Y. Lei, and J. Lin, (2017). A recurrent neural network based health indicator for remaining useful life prediction of bearings. Neurocomputing, vol. 240, pp. 98-109, doi: 10.1016/j.neucom.2017.02.045.

[28] LeCun Y, Bottou L, Bengio Y and Haffner P. (1998). Gradient-based learning applied to document recognition Proc. IEEE 86 2278-324

[29] Lei, Y., He, Z., \& Zi, Y. (2011). EEMD method and WNN for fault diagnosis of locomotive roller bearings. Expert Systems with Applications, 38(6), 7334-7341.

[30] Li, S., Xin, Y., Li, X., Wang, J., \& Xu, K. (2019, May). A Review on the Signal Processing Methods of Rotating Machinery Fault Diagnosis. In 2019 IEEE 8th Joint International Information Technology and Artificial Intelligence Conference (ITAIC) (pp. 1559-1565). IEEE.

[31] Lijun, Z.; Kai, L.; Yufeng,W.; Zachary, B.O. (2018). Ice Detection Model of Wind Turbine Blades Based on Random Forest Classifier. Energies, 11, 2548.

[32] Lu C, Wang Z. Y, Qin W. L. and Ma J. (2017). Fault diagnosis of rotary machinery components using a stacked denoising autoencoder-based health state identification Signal Process. 130 377-88

[33] M. A. Awadallah, S. Member, M. M. Morcos, and S. Member, (2003). Application of AI Tools in Fault Diagnosis of Electrical Machines and Drives - An Overview," IEEE Trans. ENERGY Convers., vol. 18 , no. 2, pp. 245-251.

[34] M. M. Ettefagh, M. Ghaemi, and M. Yazdanian Asr (2014). Bearing fault diagnosis using hybrid genetic algorithm K-means clustering," INISTA 2014 - IEEE Int. Symp. Innov. Intell. Syst. Appl. Proc., vol. 978, no. 4799-3020, pp. 84-89, doi: 10.1109/INISTA.2014.6873601.

[35] Ma M., Sun C. and Chen X. (2017). Discriminative deep belief networks with ant colony optimization for health status assessment of machine IEEE Trans. Instrum. Meas. 66 3115-25

[36] Meng Z., Zhan X, Li J. and Pan Z. (2018). An enhancement denoising autoencoder for rolling bearing fault diagnosis Measurement

[37] Nerella, M. J., \& Ratnam, C. (2018). Fault Diagnosis of a Rolling Element Bearings Using Acoustic Condition Monitoring and Artificial Neural Network Technique. International Research Journal of Engineering and Technology (IRJET), 5(3).

[38] Raúl, P.; Jordi, F.; Jordi, C.R. (2019). Predicting Energy Generation Using Forecasting Techniques in Catalan Reservoirs.Energies, 12, 1832.

[39] Robert B Randall (2004). State of the art in monitoring rotating machinery-part 1. Sound and vibration, 38(3):14-21.

[40] S. Min, B. Lee, S. Yoo, (2017). Deep learning in bioinformatics. Briefings Bioinf 18, 851-869

[41] S. Suh, H. Lee, J. Jo, P. Lukowicz, and Y. O. Lee, (2019). Generative oversampling method for imbalanced data on bearing fault detection and diagnosis. Appl. Sci., vol. 9, No. 4, doi: 10.3390/app9040746.

[42] Schmidhuber J. (2015). Deep learning in neural networks: an overview Neural Netw. 61 85-117

[43] Shao H., Jiang H., Wang F. and Wang Y. (2017). Rolling bearing fault diagnosis using adaptive deep belief network with dual-tree complex wavelet packet ISA Trans. 69 187-201

[44] Shao H., Jiang H., Zhang X. and Niu M. (2015). Rolling bearing fault diagnosis using an optimization deep belief network Meas. Sci. Technol. 26115002

[45] Shenfield, A., \& Howarth, M. (2020). A novel deep learning model for the detection and identification of rolling element-bearing faults. Sensors, 20(18), 5112.

[46] Singh J, Darpe A. K. and Singh S. P. (2019). Bearing remaining useful life estimation using an adaptive data driven model based on health state change point identification and $\mathrm{K}$-means clustering Meas. Sci. Technol. 31085601.

[47] T. W. Rauber, F. D. A. Boldt, and F. M. Varejão ( 2015). Heterogeneous Feature Models and Feature Selection Applied to Bearing Fault Diagnosis," IEEE Trans. Ind. Electron., vol. 62, No. 1, pp. 637-646,.

[48] Viola, J., Chen, Y., \& Wang, J. (2021). FaultFace: Deep convolutional generative adversarial network (DCGAN) based ball-bearing failure detection method. Information Sciences, 542, 195-211.

[49] W. Abed, S. Sharma, R. Sutton, and A. Motwani (2015). A Robust Bearing Fault Detection and Diagnosis Technique for Brushless DC Motors Under Non-stationary Operating Conditions. J. Control. Autom. Electr. Syst., vol. 26, No. 3, pp. 241-254., doi:10.1007/s40313-015-0173-7. 
[50] Wang, H., Yu, Z., \& Guo, L. (2020, April). Real-time online fault diagnosis of rolling bearings based on KNN algorithm. In Journal of Physics: Conference Series (Vol. 1486, No. 3, p. 032019). IOP Publishing.

[51] Wang, J., Wang, D., Wang, S., Li, W., \& Song, K. (2021). Fault Diagnosis of Bearings Based on Multi-Sensor Information Fusion and 2D Convolutional Neural Network. IEEE Access, 9, 2371723725.

[52] Wenyi, L., Zhenfeng, W., Jiguang, H., \& Guangfeng, W. (2013). Wind turbine fault diagnosis method based on diagonal spectrum and clustering binary tree SVM. Renewable Energy, 50, 1-6.

[53] Xia M., Li T., Liu L., Xu L. and de Silva C. W. (2017) Intelligent fault diagnosis approach with unsupervised feature learning by stacked denoising autoencoder IET Sci. Meas. Technol. 11 687-95

[54] Xu F. and Tse P. W. (2019). Combined deep belief network in deep learning with affinity propagation clustering algorithm for roller bearings fault diagnosis without data label J. Vib. Control 25 473-82

[55] Y. LeCun, Y. Bengio, G. Hinton (2015). Deep learning. Nature 521(7553), 436-444

[56] Y. O. Lee, J. Jo, and J. Hwang, (2017). Application of deep neural network and generative adversarial network to industrial maintenance: A case study of induction motor fault detection. Proc. - 2017 IEEE Int. Conf. Big Data, Big Data, vol. 2018-Januar, pp. 3248-3253, doi: 10.1109/BigData.2017.8258307.

[57] Y. Xie and T. Zhang, (2018). Imbalanced Learning for Fault Diagnosis Problem of Rotating Machinery Based on Generative Adversarial Networks. Chinese Control Conf. CCC, vol., pp. 60176022, 2018-July, doi: 10.23919/ChiCC.2018.8483334.

[58] Zhang C., Lim P., Qin A. K. and Tan K. C. (2017). Multiobjective deep belief networks ensemble for remaining useful life estimation in prognostics IEEE Trans. Neural Netw. Learn. Syst. 282306 18

[59] Zhang K., Li Y., Scarf P. and Ball A. (2011) Feature selection for high-dimensional machinery fault diagnosis data using multiple models and radial basis function networks Neurocomputing 74 2941-52

[60] Zhang, B., Li, W., Hao, J., Li, X. L., \& Zhang, M. (2018). Adversarial adaptive 1-D convolutional neural networks for bearing fault diagnosis under varying working condition. arXiv preprint arXiv:1805.00778.

[61] Zhang, J., Yi, S., Liang, G. U. O., Hongli, G. A. O., Xin, H. O. N. G., \& Hongliang, S. O. N. G. (2020). A new bearing fault diagnosis method based on modified convolutional neural networks. Chinese Journal of Aeronautics, 33(2), 439-447.

[62] Zhang, S., Zhang, S., Wang, B., \& Habetler, T. G. (2020). Deep learning algorithms for bearing fault Diagnostics-A comprehensive review. IEEE Access, 8, 29857-29881.

[63] Zhang, Y., Xing, K., Bai, R., Sun, D., \& Meng, Z. (2020). An enhanced convolutional neural network for bearing fault diagnosis based on time-frequency image. Measurement, 157, 107667.

[64] Zhao, K., Jiang, H., Wang, K., \& Pei, Z. (2021). Joint distribution adaptation network with adversarial learning for rolling bearing fault diagnosis. Knowledge-Based Systems, 222, 106974. 